\title{
The stability of the MEMS 2D mirror's operating point in terms of eye tracking systems
}

\author{
Mateusz Pomianek ${ }^{* 1}$ Marek Piszczek ${ }^{1}$, Marcin Maciejewski ${ }^{1}$ and Piotr Krukowski ${ }^{2}$ \\ ${ }^{1}$ Institute of Optoelectronics, Military University of Technology, 2 Kaliski Str., 00-908 Warsaw, \\ ${ }^{2}$ RemmedVR sp. z o.o., 47/10 Domaniewska Str., 02-672 Warsaw
}

Received May 14, 2020; accepted June 27, 2020; published June 30, 2020

\begin{abstract}
This paper describes research on the stability of the MEMS mirror for use in eye tracking systems. MEMS mirrors are the main element in scanning methods (which is one of the methods of eye tracking). Due to changes in the mirror pitch, the system can scan the area of the eye with a laser and collect the signal reflected. However, this method works on the assumption that the inclinations are constant in each period. The instability of this causes errors. The aim of this work is to examine the error level caused by pitch instability at different points of work.
\end{abstract}

Eye tracking systems are now largely based on vision solutions $[1 \div 2]$. In these methods, the eye pupil position is determined on the basis of image analysis (from the camera observing the eye). Afterwards, the user's point of gaze is estimated using this information [3]. However, the accuracy of this method mainly relies on image resolution [4]. On the other hand, higher resolution means that more processing power is needed for image analysis (to maintain the same operating speed) [5]. In the case of mobile eye tracking systems, the processing power is very limited. Systems based on image analysis must work with low-resolution cameras to maintain smooth operation [6]. This, in turn, causes such solutions to have relatively low accuracy. An alternative in such situations may be the use of scanning methods $[5 \div 6]$. Such systems illuminate the eye area with a laser beam and collect the reflected signal. Based on the difference in signal intensity, the pupil's circumference and thus its position is estimated $[9 \div 10]$. The main element of these systems is the MEMS mirror. Due to changes in its pitch, the system can scan the area of the eye $[11 \div 12]$.

Comparing the two methods, a matrix is processed in the image analysis method (from several hundred thousand to several million pixels), but in the scanning method only a single vector is analyzed (several thousand points). The application of the scanning method in the eye tracking system allows obtaining high accuracy with low computing power. An example of an OPUS Microsystems MEMS mirror is shown in Fig. 1.

\footnotetext{
*e-mail: mateusz.pomianek@wat.edu.pl
}

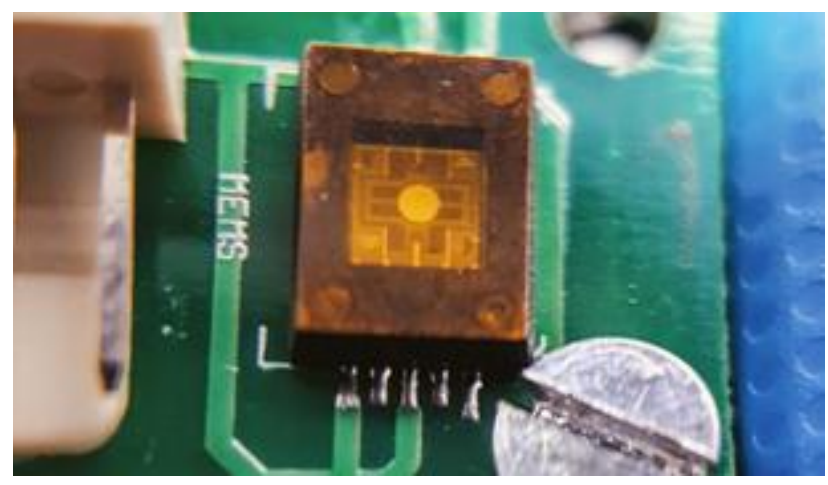

Fig. 1. OP-6111 MEMS mirror by OPUS Microsystems

The operation of the scanning method, however, is based on the assumption that the MEMS mirror pitch is proportional to the control signal in each period. If there is an inconsistency here, errors arise that cause the pupil position to be miscalculated at the very beginning. The aim of this work is to determine how the stability of the mirror's pitch affects errors in the estimation of the pupil position.

The research was carried out on a MEMS mirror from OPUS Microsystems (model OP-6111). Its practical operating frequency range is $43763 \div 44247 \mathrm{~Hz}$ for the high-speed axis and $3265 \div 3600 \mathrm{~Hz}$ for the slow axis. The pitch in each axis of the mirror is directly proportional to the operating frequency - fast axis $\pm 20^{\circ}$, slow axis $\pm 15^{\circ}$. As the mirror operates in bi-resonant mode, the curve drawn by the laser in the area of the eye can be described by Lissajous figures. A $5 \mathrm{~mW}$ laser diode working at $650 \mathrm{~nm}$ wavelength has been used as a source for measurements.

In order to determine the operating instability of the MEMS mirror, measurements were taken at the maximum tilt frequency $\left(15^{\circ}\right)$ of the high-speed axis - $43383 \mathrm{~Hz}$. Two measurement points were adopted. The first one was the point of maximum tilt, while the second one was the mirror's base position $\left(0^{\circ}\right)$. The system, thanks to synchronization with the laser, emitted a pulse in the pitch, which was determined by the specified delay of the control signal (zero delay for maximum pitch, half period for zero pitch). For each measurement, 25000 samples 
were collected. The measurements were taken in two independent series, between which the system was reconnected, calibrated, and adjusted. The optical path length was $10 \mathrm{~m}$. A camera with a resolution of $1920 \times 1080$ pixels was used as a detector. The reading accuracy was $4.87 \cdot 10^{-4}$ degrees (1.7 arc seconds). The block diagram of the measuring station is shown in Figure 2. In each sample the pitch was measured in the main axis (the one on which the mirror works in each measurement) and the side axis (on which there should be no motion).

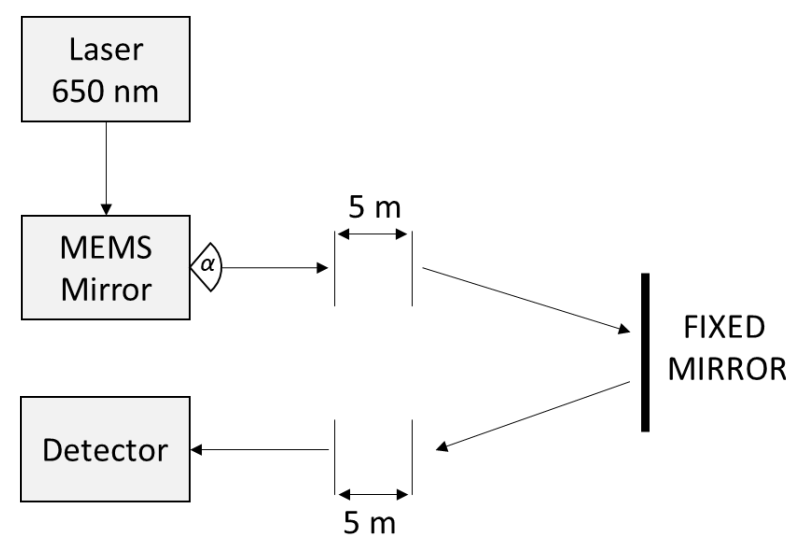

Fig. 2. Measuring station scheme.

MATLAB (v. R2019b) from MathWorks was used to analyze the results. The software analyzed each signal sample and then determined the pitch based on the position of the point.

The results showed the difference between the stability at the base position of the mirror and a $15^{\circ}$ pitch. The test results for both settings are presented in Table 1.

Tab. 1. Standard deviation of pitch stability.

\begin{tabular}{|l|c|c|}
\hline & $0^{\circ}$ & $15^{\circ}$ \\
\hline Main axis & $16.7^{\prime \prime}$ & $68.7^{\prime \prime}$ \\
\hline Side axis & $7.7^{\prime \prime}$ & $11^{\prime \prime}$ \\
\hline
\end{tabular}

Diagrams of the pitch stability (for main and side axes) in the 4000 sample range are shown in Figure 3. The blue color represents the maximum tilt. Base pitch was marked with red.
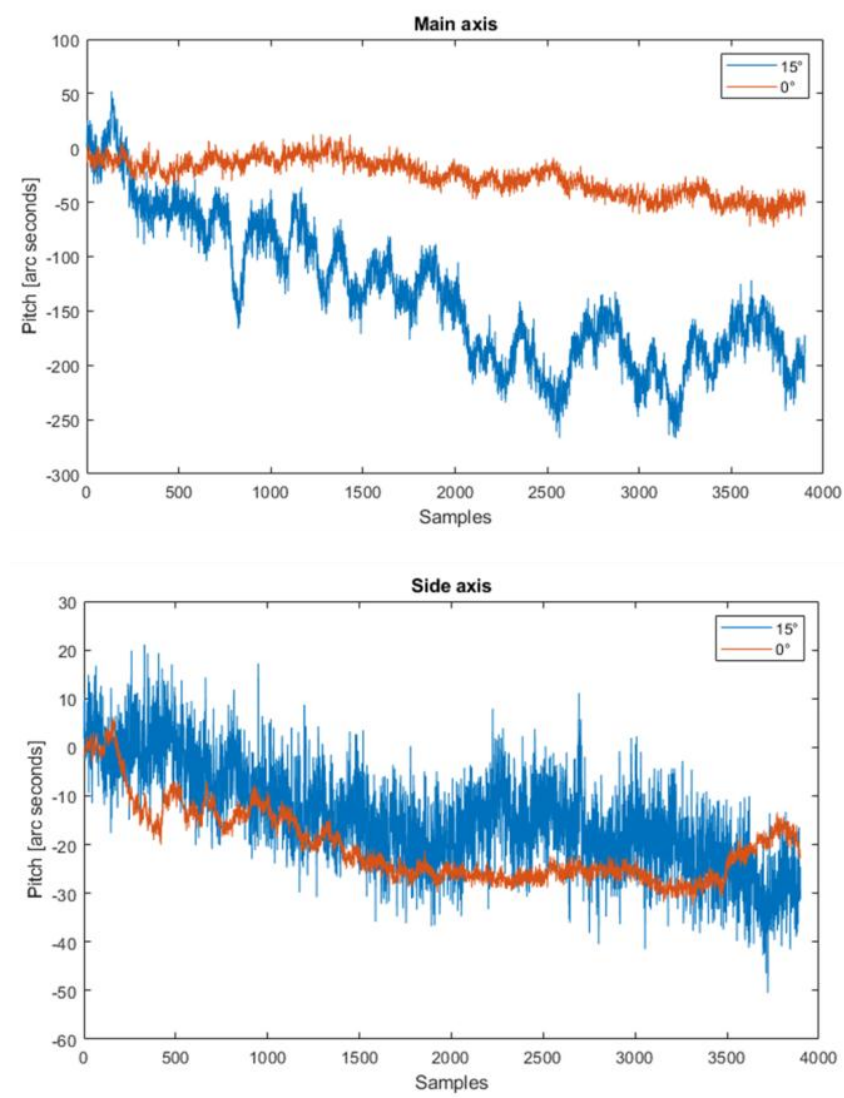

Fig. 3. MEMS mirror stability charts

Short-term pitch stability was also examined (samples < 1000). The standard deviation was 4.61 " for the main axis and $1.45 "$ for the side axis.

Due to the drift that was observed in the results, the analysis of the signal was also carried out with its omission. It was determined individually for each signal and corrected. The standard deviation of the results not including a drift is shown in Table 2 below.

Tab. 2. Standard deviation of pitch stability without a drift.

\begin{tabular}{|l|c|c|}
\hline & $0^{\circ}$ & $15^{\circ}$ \\
\hline Main axis & $9.5^{\prime \prime}$ & $58.3^{\prime \prime}$ \\
\hline Side axis & $5.4^{\prime \prime}$ & $8^{\prime \prime}$ \\
\hline
\end{tabular}

In order to determine the optimum operating point of the MEMS mirror, it was necessary to study the stability of its operation for individual axle deflections. The standard deviation of the full length was, on average, five times greater at full pitch than at no pitch. In the short term, it was also higher for maximum pitch, but only 3 times higher.

There was a drift in the signal. It occurred in every series and measurements. The drift in the maximum case caused the initial value to shift by 0.2 degrees. The system was stiffened, and its configuration was changed between the series, and yet a drift occurred in both series. 
Therefore, an environmental impact cannot be excluded, but its probability is low. If the drift was caused by the mechanical system of the MEMS mirror, it shows this additional phenomenon which must be taken into account when designing systems based on this device.

The instability of the MEMS mirror tilting affects the accuracy of pupil position estimation. Determined maximum value $68.7^{\prime \prime}\left(0.02^{\circ}\right)$ may cause a relative error of $2 \cdot 10^{-3}$, while for results with a corrected drift (58.3") it was $1.7 \cdot 10^{-3}$. For a short-term periodical stability of $4.61 "$, the error was $1.33 \cdot 10^{-4}$. The effect of the mirror tilt stability on the error of pupil position estimation is linear in the examined range and is shown (within a range of up to 100 arc second) in Fig. 4.

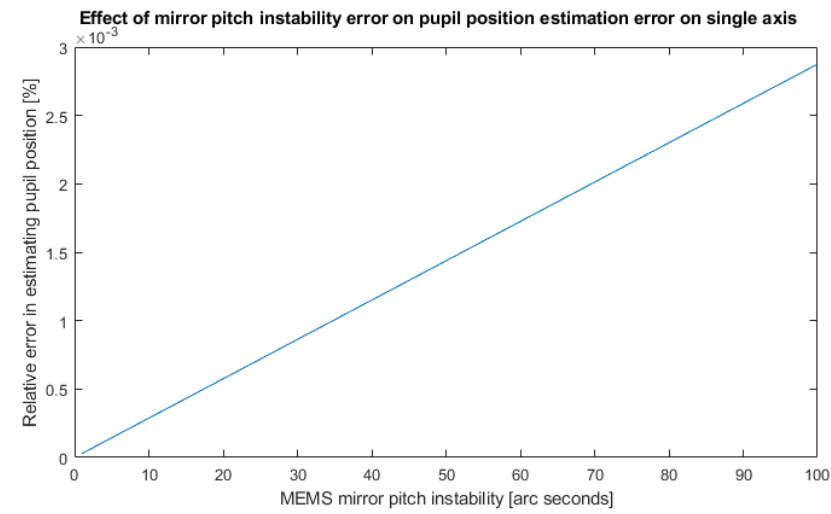

Fig. 4. Effect of mirror pitch instability error on pupil position estimation error.

In this study the pitch stability of the OPUS Microsystems MEMS mirror (model OP-6111) was examined. The results showed that it depends on the mirror's placement during operation. The highest stability was observed when the mirror was in the middle point $(0$ degrees) and the lowest when it was in the maximum pitch (15 degrees).

The standard deviation of $15^{\circ}$ tilt was 5.13 times higher than at $0^{\circ}$. Short-term stability also showed this dependence. In this case, the standard deviation of $15^{\circ}$ tilt was on average 3.2 times higher. On the basis of these results, direct correlation between the pitch and stability can be deduced, which can be used as a basis for further studies. A potential direction of future research in this area could also be to investigate the aspect of an emerging drift and its causes.

A relative error in estimating pupil position $2 \cdot 10^{-3}$ is acceptable for eye tracking applications. It should be borne in mind in terms of scientific measurement systems. However, for entertainment applications this has a small or negligible impact.
This work was supported by Project "RemmedVR device for home vision therapy".

\section{References}

[1] W. Fuhl, M. Tonsen, A. Bulling, E. Kasneci, Mach. Vis. Appl. 27(8), 1275 (2016); doi: 10.1007/s00138-016-0776-4.

[2] X. Wang, S. Koch, K. Holmqvist, M. Alexa, ACM Trans. Graph. 37(6), 1 (2018); doi: $10.1145 / 3272127.3275094$.

[3] X. Xiong, H. Xie, Proc. 2014 Zo. 1 Conf. Am. Soc. Eng. Educ., ASEE Zo. 1 2014, no. Dmd, 2014, doi: 10.1109/ASEEZone1.2014.6820679.

[4] E. Pengwang, K. Rabenorosoa, M. Rakotondrabe, N. Andreff, Micromachines 7(2), 24 (2016); doi: 10.3390/mi7020024.

[5] J.P. Giannini, A.G. York, H. Shroff, PLoS One 12(10), 1 (2017); doi: 10.1371/journal.pone.0185849.

[6] C. Hennessey, B. Noureddin, P. Lawrence, Eye Track. Res. Appl. Symp. 2005, 87 (2005); doi: 10.1145/1117309.1117349.

[7] C.H. Morimoto, M.R.M. Mimica, Comput. Vis. Image Underst. 98(1), 4 (2005); doi: 10.1016/j.cviu.2004.07.010.

[8] S.T.S. Holmström, U. Baran, H. Urey, J. Microelectromechanical Syst. 23(2), 259 (2014); doi: 10.1109/JMEMS.2013.2295470.

[9] C.W. Cho, ETRI J. 34(4), 542 (2012); doi: 10.4218/etrij.12.0111.0193.

[10] T. Santini, W. Fuhl, E. Kasneci, Comput. Vis. Image Underst. 170, 40 (2018); doi: 10.1016/j.cviu.2018.02.002.

[11] O. Solgaard, A.A. Godil, R.T. Howe, L.P. Lee, Y.A. Peter, H. Zappe, $\begin{array}{llll}\text { J. Microelectromechanical } & \text { Syst. } & \text { 23(3), } 517 \quad \text { (2014); doi: }\end{array}$ 10.1109/JMEMS.2014.2319266.

[12] J. Wang, G. Zhang, Z. You, J. Micromechanics Microengineering 29(3), 035005 (2019); doi: 10.1088/1361-6439/aaf943. 